\title{
Clinical indicators of mycobacteraemia in adults admitted to hospital in Blantyre
}

\author{
DK Lewis', RPH Peters', MJ Schijffelen', GRF Joaki², AL Walsh'³, JG Kublin", J Kumwenda', S Kampondeni', ME Molyneux',3, EE Zijlstra'
}

Departments of Medicine', Microbiology ${ }^{2}$ and Community Health ${ }^{4}$, University of Malawi College of Medicine, Blantyre; ${ }^{3}$ Malawi-Liverpool-Wellcome Trust Clinical Research Programme, College of Medicine, Blantyre For correspondence: Dr DK Lewis, Email - mariadavid@callaghanlewis. fsnet.co.uk

The aims of the study were to measure the prevalence and outcome of mycobacteraemia in febrile hospitalised adults; to determine what proportion could be identified using routine methods; to assess clinical indicators of mycobacteraemia and the usefulness of a diagnostic trial of anti-TB treatment. We prospectively examined adults with fever or a history of fever admitted to adult medical wards of QECH, Blantyre. All had blood cultured for bacteria and mycobacteria, chest x-ray and sputum smears. M. tuberculosis was the commonest blood isolate, affecting 57 of 344 patients (17\%). In $44(77 \%)$ patients with mycobacteraemia, TB was identified using routine investigations; in only $6(11 \%)$ it was not suspected. Strong clinical indicators of mycobacteraemia were anaemia, HIV seropositivity, cough, chronic fever, and a clinical diagnosis of AIDS on the day of admission. Of nine patients selected for a therapeutic trial of TB treatment, six had mycobacteraemia, of whom five died during the trial. Mortality on short course chemotherapy, on the TB ward after one month, was similar whether patients had mycobacteramia ( $21 \%)$ or not $(32 \%)$. TB can be identified with routine methods in most patients with mycobacteraemia. If treated, mycobacteraemia has as good an early outcome as TB without mycobacteraemia. Strengthening of basic facilities is likely to improve detection and treatment of mycobacterial disease.

\section{Introduction}

Mycobacteraemia was described as a feature of tuberculosis eighty years ago.[1] Only in the era of AIDS has it become recognised as common.[2-4] Recent studies where HIV and tuberculosis abound have shown that Mycobacterium tuberculosis may be the commonest organism in blood stream infection (BSI), with prevalence among febrile adult inpatients ranging from 9 to $12 \%$.[5-8] Mycobacterial culture is slow, and many patients will die or be discharged before culture is positive. [5,911.] Culture is expensive and not routinely available in subSaharan Africa. Mortality in those who do not receive treatment may be as much as $80 \% .[5,12]$ None of these published studies had sufficient clinical information to determine whether tuberculosis could be diagnosed in these patients without using blood cultures; very few patients were investigated by sputum examination or chest $x$-ray. $[5-8,12]$ It has been suggested that better microbiology services in developing countries would improve detection of tuberculosis (TB) [7], but this would be unnecessary if the available, and cheaper, investigations would be equally or more useful clinically.

Mycobacteraemia has been associated with chronic symptoms of cough, fever and weight loss [5-8], with oral thrush [8], anaemia $[6,12]$, low CD4+ or total lymphocyte counts $[2,12]$ and hepatosplenomegaly.[13] The clinical spectrum may range from acute fulminating infection, in which patients may die before tubercles are seen on X-ray, through to a chronic infection in miliary disease [14]. It is not known where along this spectrum most patients in sub-Saharan Africa lie, nor in what proportion the diagnosis may be made using existing routine methods alone. Most patients with disseminated TB become afebrile within two weeks of starting treatment.[13] Where TB is suspected in unexplained fever, a therapeutic trial of specifically anti-tuberculous drugs (isoniazid, pyrazinamide and ethambutol) has been suggested.[15,16]

We performed a prospective study of blood-stream infection
(BSI) in febrile adult admissions to our hospital, and investigated all patients for TB using methods routinely available in most hospitals in sub Saharan Africa. We measured the prevalence and outcome of mycobacteraemia, and the proportion of mycobacteraemic cases that could be identified using routine methods. We assessed the clinical indicators of mycobacteraemia, and the usefulness of a diagnostic trial of anti-TB treatment.

\section{Methods}

Queen Elizabeth Central Hospital is the main provider of secondary care for Blantyre district. This study took place between 15 February 2000 and 2 May 2000. We recruited patients on twenty-three week days, of 24 hours, randomly chosen in advance. All patients admitted to the adult medical wards on the specified days (excluding direct admissions to the TB ward) were assessed. Patients were eligible for the study if they had an axillary temperature $\geq 37.4 \mathrm{deg} \mathrm{C}$, or were in shock (pulse $\geq 120$ beats/minute and systolic blood pressure $<100 \mathrm{~mm} \mathrm{Hg}$ ), or if they gave a history of a high fever within the last four days; and if they consented. We included afebrile patients with a recent history of fever, because mycobacterial blood cultures are often positive even if not taken at the time of fever.[17] Approval for the study was obtained from the Research Committee of the College of Medicine, University of Malawi.

A study clinician conducted a standardised history and clinical examination. The optic fundi were examined by an ophthalmologist. Blood was drawn aseptically for bacterial and mycobacterial culture, full blood count, film for malaria parasites, and HIV tests. Each patient was given three bottles for sputum production. Chest $x$-ray was taken within 24 hours. Other investigations were performed when appropriate. Patients were managed according to standard protocols. All patients were reviewed by a physician within 24 hours (DKL, JK, EEZ, JGK, or MEM), and physical examination was repeated. They were reviewed daily 
while inpatients. On the fourth day bacterial blood culture and blood film were repeated if fever was present. Patients whose mycobacterial culture became positive after discharge were followed up at home if not already receiving TB treatment, and outcome one month after admission was established for all with mycobacteraemia.

For the purposes of clinical management, tuberculosis was diagnosed by finding any of: acid-alcohol-fast bacilli in sputum or lymph node aspirate; caseous lymph node aspirate; 'classical' chest x-ray changes (cavities, pulmonary fibrosis, upper lobe or bilateral infiltrates, or pleural effusion not otherwise explained); 'non-classical' chest x-ray changes (other infiltrates or intrathoracic adenopathy) together with chronic ( $\geq 1$ month) cough or chronic fever; miliary pattern on chest $\mathrm{x}$-ray; pericardial effusion confirmed by ultrasound; or TB meningitis (chronic headache plus cerebrospinal fluid leucocyte count $>200 / \mathrm{ml}$ with lymphocytes $\geq 80 \%$ ). Chest $\mathrm{x}$-rays $(\mathrm{CXR})$ were reviewed by an experienced radiologist (SK) blind to clinical details.

\section{Trial of TB Treatment}

Patients with an identifiable clinical diagnosis, a positive blood film for malaria, or a bacterial growth from blood culture were treated accordingly. Patients with a clinical or bacteriological diagnosis of $\mathrm{TB}$ were treated according to national policy. Disseminated TB was considered a possible diagnosis in patients with unexplained persistent fever. Those with negative bacterial blood culture, negative blood film and sputum smear, and a chest $\mathrm{x}$-ray not suggestive of $\mathrm{TB}$ were given broad spectrum antibiotics (gentamicin, plus penicillin or chloramphenicol) for five days. Any patient still febrile after five days received a diagnostic trial of specific anti-TB drugs - daily isoniazid, pyrazinamide and ethambutol. If the clinical condition had improved after three weeks the patient was assumed to have TB and was given short course chemotherapy.

\section{Laboratory Methods}

Full Blood Count was performed with a Coulter Counter. HIV testing was with Abbot Determine (Dainabot Company, Tokyo, Japan) and Unigold (Trinity Biotech, Bray, Ireland); patients with a positive result in both tests were regarded as HIV positive. Sputum smears were stained with Ziehl Neelsen. For the routine cultures $5 \mathrm{mls}$ blood in a single blood culture bottle $(50 \mathrm{mls}$ Brain Heart Infusion Broth containing sodium polyanethol sulphonate, E\&O Laboratories, UK) was incubated overnight at $37^{\circ} \mathrm{C}$ before venting. Cultures were examined macroscopically every day, followed by Gram staining if turbid or haemolysed. Isolates were identified according to standard techniques, including seroagglutination and biochemical tests. Mycobacterial blood cultures were performed using Bactec 13A blood culture bottles (Becton Dickinson, Oxford UK), examined manually. Once a week for a maximum of 8 weeks, a smear was prepared and stained with auramine-phenol. Positive cultures which showed typical cording were reported as presumptive Mycobacterium tuberculosis, while those which did not cord were reported as Mycobacterium species. Positive mycobacterial cultures underwent DNA extraction, then PCR for Mycobacterium specific DNA and $M$. tuberculosis specific DNA.

\section{Statistical Methods}

Data were double-entered into EpiInfo version 6.04b (CDC, USA; WHO, Switzerland), validated, and analysed with Stata 6.0 (Stata Corporation, Texas 77840, USA). Categorical variables were compared with $\mathrm{chi}^{2}$, or Fisher's exact test.
Continuous data were compared with Mann-Whitney rank sum test, if skewed. Odds Ratios were calculated and adjusted with logistic regression.

\section{Results}

There were 637 admissions on study days, of whom $367(58 \%)$ were eligible for the study. Twelve patients declined, two were transferred to another hospital, and one was missed by the study team. Eight patients, on TB treatment at the time of admission, were excluded. In all 344 patients entered the study, 151 men and 193 women. All except 17, with a history of recent fever, were febrile on admission. The mean age was 31.5 years (range 15-64). 285 (83\%) patients were HIV sero-positive, and 156 $(46 \%)$ met the expanded WHO case definition for AIDS surveillance on the day of admission.[18]

Table 1. Microbial causes of Bloodstream Infections in HIV positive and negative patients

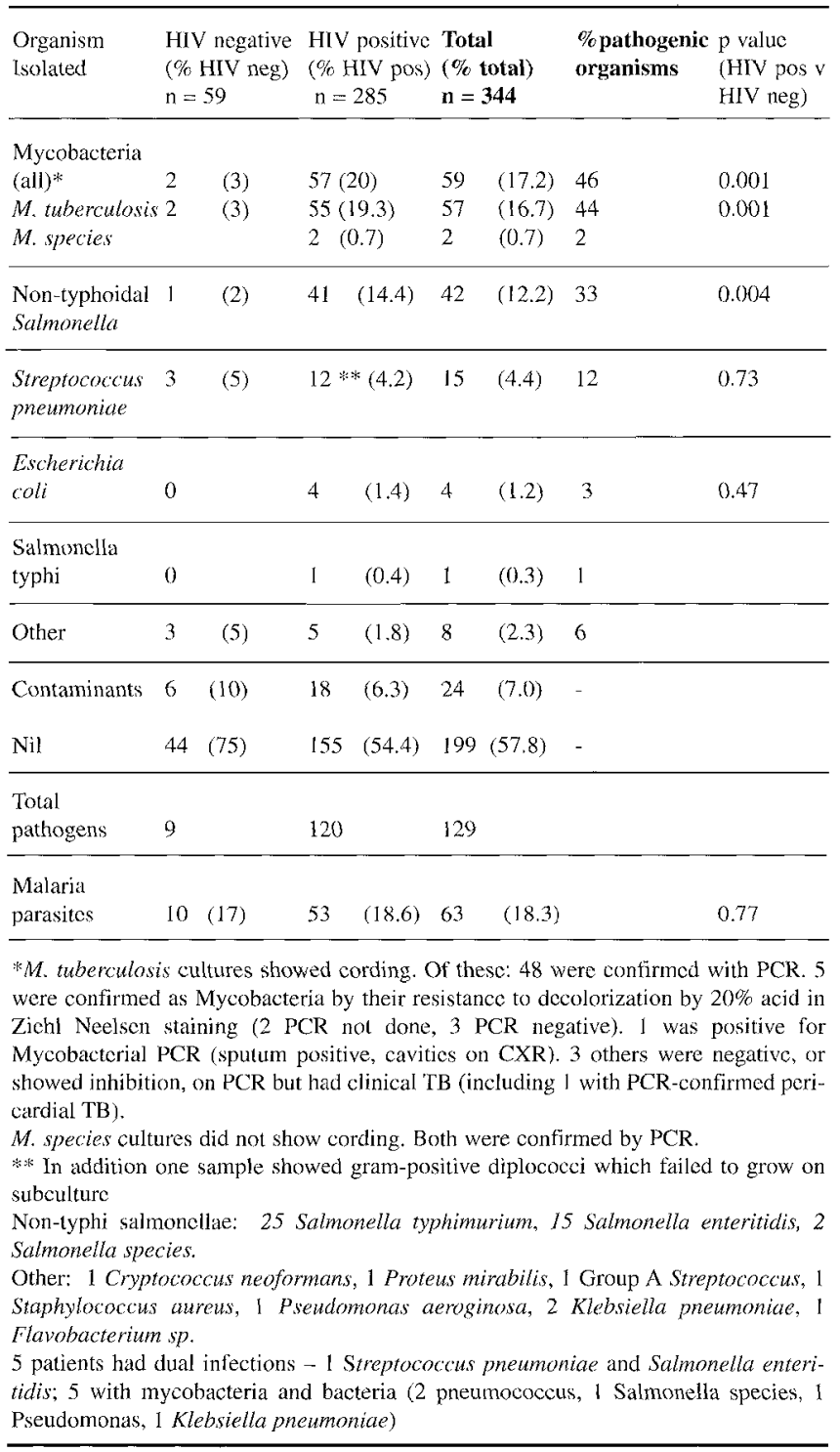

Table 1 shows the blood culture results. The commonest infecting organsism was $M$. tuberculosis, affecting $17 \%$ of all study patients, followed by non-typhi salmonellae. Mycobacteria were confirmed as described in Table 1. Of patients admitted afebrile the proportion with bacteraemia $(18 \%)$ and mycobacteraemia (12\%) was similar to those febrile on admission.

TB was diagnosed by routine methods in 103 (30\%) study patients,of whom $97(94 \%)$ were HIV positive. Of all diagnosed with TB, $44(43 \%)$ had mycobacteraemia, while among the 241 
not diagnosed with TB, $M$. tuberculosis BSI or 'mycobacteraemia' was identified in $13(5.4 \%)$, and non-tuberculous mycobacteraemia in $2(0.8 \%)$. Of 57 patients with mycobacteraemia, $44(77 \%)$ had TB diagnosed with routine methods. The methods used are shown in Table 2:

Table 2. Routine methods of diagnosing TB

\begin{tabular}{|c|c|c|c|c|}
\hline \multirow[t]{2}{*}{ Diagnostic method } & \multicolumn{2}{|c|}{$\begin{array}{l}\text { All patients } \\
\text { diagnosed with TB }\end{array}$} & \multicolumn{2}{|c|}{$\begin{array}{l}\text { Patients with } \\
\text { M. tubcrculosis BSI }\end{array}$} \\
\hline & Number & $(\%)$ of 103 & Number & $(\%)$ of 57 \\
\hline \multicolumn{5}{|l|}{ Confirmed Sputum } \\
\hline AFB positive & 28 & $(27)$ & 18 & (32) \\
\hline $\begin{array}{l}\text { Lymph node } \\
\text { aspirate caseous }\end{array}$ & 13 & (13) & 5 & (9) \\
\hline $\begin{array}{l}\text { Total bacteriologically } \\
\text { confirmed (not including } \\
\text { blood culture) }\end{array}$ & 38 & (37) & 21 & $(37)$ \\
\hline \multirow{3}{*}{$\begin{array}{l}\text { Classical CXR } \\
\text { non-classical CXR } \\
\text { Miliary CXR }\end{array}$} & 47 & (46) & 17 & $(30)$ \\
\hline & 35 & (34) & 18 & (32) \\
\hline & 11 & (11) & 9 & (16) \\
\hline Total CXR & 93 & $(90)$ & 44 & (77) \\
\hline \multicolumn{5}{|l|}{ Pericardial effusion } \\
\hline on ultrasound & 4 & (4) & 1 & (2) \\
\hline $\mathrm{TB}$ meningitis & 3 & (3) & 1 & (2) \\
\hline \multicolumn{5}{|c|}{$\begin{array}{l}\text { * Bacteriological confirmation includes sputum AFB positive, lymph node aspirate } \\
\text { and pleural fluid culture positive }\end{array}$} \\
\hline \multicolumn{5}{|c|}{$\begin{array}{l}\text { *: CXRs suggestive of TB were classified as 'classical' (showing cavities, pulmonary } \\
\text { fibrosis, upper lobe or bilateral infiltrates, or pleural effusion not explained by } \\
\text { another disease), 'miliary', or 'non-classical' (other infiltrates or intrathoracic } \\
\text { adenopathy together with chronic congh or fever). Fifteen patients had their } \\
\text { pleural effusion aspirated, of which } 8 \text { were TB culture positive } \\
\text { ( } 3 \text { mycobacteraemic). All except one patient (with TB meningitis) had CXR. }\end{array}$} \\
\hline \multicolumn{5}{|c|}{$\begin{array}{l}\text { All had sputum examination except } 7 \text { with mycobacteracmia and } 3 \text { without. } \\
\text { Lymph node aspirates were from: } 6 \text { axilla, } 5 \text { neck, } 1 \text { inguinal, } 1 \text { abdominal. } 5 \text { of these } \\
\text { were confirmed } A F B \text { positive. }\end{array}$} \\
\hline Note that some patients were & diagnosed & oy more than & e method. & \\
\hline
\end{tabular}

one third ( $41 \%$ of those clinically diagnosed) were sputum pos itive and all of those who had TB diagnosed clinically had CXRs suggestive of TB. Of the 103 patients diagnosed with TB, 61 $(59 \%)$ had bacteriologically proven TB (sputum or lymph node aspirate AFB positive, pleural fluid culture positive, or subsequent blood culture positive). This rose to $66(64 \%)$ if those with pericardial effusion on ultrasound, or a miliary pattern on CXR were included. Only $12(12 \%)$ patients were diagnosed solely on the basis of a non-classical CXR plus symptoms.

Thirteen individuals (23\%) with mycobacteraemia were not recognised as having $\mathrm{TB}$ until blood culture became positive. Of these: four had been started on a therapeutic trial of TB treatment; three had clinical pneumonia (one died within a few days, two improved); three gave a history of chronic fever but improved on the ward; one absconded while still febrile; one started antibiotics for chronic cough but did not return for follow up; and one gave a three day history of cough which improved on the ward. Thus 7 were strongly suspected of TB (4 trial of treatment, $1 \mathrm{djed}, 2$ did not attend for review), and 6 (11\% those with mycobacteraemia) were truly undetectable with routine methods. Two of these were found alive and treated with short course chemotherapy.

Two patients had $M$. species BSI, both of whom had a short history of cough and clinical picture of pneumonia: one died on Day 5 , and one improved with antibiotics.

Nine patients were started on such a trial. Six were subsequently shown to have mycobacteraemia (positive predictive value of starting a trial $67 \%$. OR $50.2,95 \%$ Cl 10 to 234 , compared with all other patients not clinically diagnosed with TB). Five had abnormal CXRs not suggestive of TB. Five died during the trial ( 3 weeks), and the sixth died after 5 weeks, having failed to return for review. Of four mycobacteraemic patients given a trial as inpatients, only one became afebrile (but died 4 days later). Of the non-mycobacteraemic patients given a trial, two died within 6 days of starting treatment, and the third requested discharge and was not traced.

Mortality on the medical wards was $19 \%$ in patients with mycobacteraemia - compared with $21 \%$ in those with other (bacterial) BSI. In patients with no BSI mortality was $8 \%$ ( $p=0.003$ ). Patients with mycobacteraemia who received short course chemotherapy on the TB ward were less likely to die during the first month than non-mycobacteraemic patients on the same treatment, but this was not statistically significant $(21 \%$ versus $32 \%, p=0.42$ ).

Table 3. Mortality one month after admission in relation to type of TB treatment, in patients with mycobacteraemia

\begin{tabular}{|c|c|c|c|}
\hline Type of treatment & $\begin{array}{l}\text { (number } \\
\text { of patients) }\end{array}$ & $\begin{array}{l}\text { Number died within } \\
1 \text { month of } \\
\text { admission }(\%)\end{array}$ & $\begin{array}{l}\text { Mortality } \\
\text { assuming } \\
\text { thosenot } \\
\text { found had } \\
\text { died }\end{array}$ \\
\hline No treatment & (14) & $7 \quad(50 \%)$ [3 not found $]$ & $71 \%$ \\
\hline $\begin{array}{l}\text { Short course } \\
\text { chemotherapy }\end{array}$ & $(28) *$ & $\begin{array}{l}8 \quad(29 \%) \text { [ } 1 \text { left ward } \\
\text { and not found] }\end{array}$ & $32 \%$ \\
\hline $\begin{array}{l}\text { Standard } \\
\text { chemotherapy }\end{array}$ & (9) & $3 \quad(33 \%)$ & $33 \%$ \\
\hline $\begin{array}{l}\text { Therapeutic trial } \\
\text { of treatment } \\
\text { All with }\end{array}$ & (6) & $5 \quad(83 \%) * *$ & $83 \%$ \\
\hline M. tuberculosis BSI & $(57)$ & $23(40 \%)$ [4 not found] & $64 \%$ \\
\hline
\end{tabular}

Table 3 shows deaths within one month of admission in patients with mycobacteraemia, according to type of treatment given. Some individuals died before treatment and some died within a few days of starting treatment. Overall $24(42 \%)$ died within one month of admission. Eleven people with (unrecognised) mycobacteraemia went home with no TB treatment. They were followed up when culture became positive. Six had died, two were alive and three could not be found.

Table 4 shows indicators of mycobacteraemia.. The strongest indicators (other than a clinical diagnosis of TB) were anaemia $(\mathrm{Hb}<10 \mathrm{~g} / \mathrm{dL})$, HIV seropositivity, cough, chronic fever, and diagnosis of AIDS on the day of admission.

307 patients had ocular examination. Four had clinically suspected choroidal TB granulomas: three of whom were mycobacteraemic with clinically disseminated TB. The fourth received a trial of treatment, although mycobacterial culture was subsequently negative. He became afebrile with treatment but died after 5 days.

Patients with mycobacteraemia were similar to non-mycobacteraemic patients who were diagnosed with TB using routine methods, in terms of age, sex, HIV status, number with AIDS on admission, body mass index, thrush, and a history of previous TB, cough, chronic fever, weight loss, night sweats, or recent antibiotics. Table 5 shows the main differences. Among patients with an eventual diagnosis of TB, those with mycobacteraemia had a shorter length of cough (median 2 weeks versus 1 month, ranksum $p=0.004$ ) than those without. Those with mycobacteraemia were less likely to have haemoptysis or a pleural effusion; and more likely to be anaemic, or sputum positive or to have miliary TB. 
Table 4. Indicators of Mycobacteraemia

\begin{tabular}{llll}
\hline Clinical indicator & $\begin{array}{l}\text { Odds Ratio } \\
(95 \% \mathrm{CI})\end{array}$ & $\mathrm{p}$ & $\begin{array}{l}\text { Adjusted Odds } \\
\text { Ratio } * * \\
(95 \% \mathrm{CI})\end{array}$ \\
\hline
\end{tabular}

\begin{tabular}{|c|c|c|c|c|c|c|}
\hline \multicolumn{7}{|l|}{ Anaemia } \\
\hline HIV positive & 6.8 & $(1.6-28.8)$ & 0.009 & 4.3 & $(0.93-19)$ & 0.061 \\
\hline Cough & 3.8 & $(1.6-8.6)$ & 0.002 & 2.8 & $(1.2-6.7)$ & 0.020 \\
\hline $\begin{array}{l}\text { Chronic fever } \\
\text { (>1 month) }\end{array}$ & 3.3 & $(1.8-5.9)$ & $<0.001$ & 2.2 & $(1-4.8)$ & 0.046 \\
\hline $\begin{array}{l}\text { AIDS on day of } \\
\text { admission (F) }\end{array}$ & 3.1 & $(1.7-5.8)$ & $<0.001$ & 3.2 & $(1.7-6.0)$ & $<0.001$ \\
\hline Body Mass Index & & & & & & \\
\hline$<17 \mathrm{~kg} / \mathrm{m} 2$ & 2.4 & $(1.3-4.4)$ & 0.003 & 1.8 & $(1-3.5)$ & 0.065 \\
\hline Pleuritic chest pain & 2.3 & $(1.2-4.1)$ & 0.007 & 1.8 & $(0.94-3.3)$ & 0.078 \\
\hline Orthopnoea & 2.0 & $(1.1-3.6)$ & 0.028 & 1.5 & $(0.82-2.9)$ & 0.179 \\
\hline Breathless on cxertion & 2.0 & $(1.1-3.8)$ & 0.029 & 1.5 & $(0.76-3.0)$ & 0.246 \\
\hline Night sweats & 2.0 & $(1.1-3.6)$ & 0.018 & 1.5 & $(0.81-2.8)$ & 0.189 \\
\hline Oral Thrush & 2.0 & $(1.1-3.7)$ & 0.028 & 1.2 & $(0.59-2.4)$ & 0.632 \\
\hline Hepatomegaly & 1.6 & $(0.9-3.1)$ & 0.123 & 1.5 & $(0.77-2.8)$ & $0.24 \mathrm{I}$ \\
\hline $\begin{array}{l}\text { Hepatomegaly } \\
\text { (excluding }\end{array}$ & & & & & & \\
\hline bacteraemia) & 1.9 & $(1.1-3.8)$ & 0.049 & 1.6 & $(0.83-3.3)$ & 0.156 \\
\hline $\begin{array}{l}\text { Given trial of TB } \\
\text { treatment (and not } \\
\text { already on } \\
\text { TB treatment)* }\end{array}$ & 50.2 & $(10.8-234)$ & .001 & 52.5 & $(9.0-303)$ & $<0.001$ \\
\hline $\begin{array}{l}\text { Sputum AFB positive } \\
\text { Miliary pattern on }\end{array}$ & 13.3 & $(5.8-31)$ & 0.001 & 11.2 & $(4.5-28.0)$ & $<0.001$ \\
\hline CXR & 24.7 & $(5.2-118)$ & $<0.001$ & 25.8 & $(5.2-128)$ & $<0.001$ \\
\hline Non-classical CXR & 6.7 & $(3.2-14.2)$ & $<0.001$ & 5.8 & $(2.6-13.1)$ & $<0.001$ \\
\hline Classical CXR & 3.0 & $(1.5-5.9)$ & 0.001 & 2.1 & $(1.0-4.4)$ & 0.045 \\
\hline $\begin{array}{l}\text { Any CXR } \\
\text { suggestive of TB }\end{array}$ & 13.9 & $(7.0-27.6)$ & $<0.001$ & 13.0 & $(6.1-27.8)$ & $<0.001$ \\
\hline
\end{tabular}

* Patients were given a therapeutic trial of ТВ treatment if they had persistent fever, negative bacterial blood culture, sputum and malaria slide, a CXR not suggestive of $T B$, and no response to broad spectrum antibiotics.

** Adjusted for age, sex, urban/rural dweller, education level reached, and diagnosis of AIDS on day of admission [181

Table 5. Differences between TB patients with and without mycobacteraemia

\begin{tabular}{llll} 
Clinical finding & $\begin{array}{l}\text { Mycobactcraemia } \\
\text { Number }(\%) \text { of } 57 \text { Number }(\%) \text { of } 59\end{array}$ & $\begin{array}{l}\text { OR for } \\
\text { mycobacteraemia } \\
{[95 \% \mathrm{Cl}]}\end{array}$ \\
\hline
\end{tabular}

\begin{tabular}{|c|c|c|c|c|c|c|}
\hline $\begin{array}{l}\text { Severe anaemia } \\
(\mathrm{Hb}<7 \mathrm{~g} / \mathrm{dL})\end{array}$ & 18 & (32) & 9 & (15) & 0.038 & $2.6\lceil 1$ to $6.3 \mid$ \\
\hline \multicolumn{7}{|l|}{ Anaemia } \\
\hline$(\mathrm{Hb}<10 \mathrm{~g} / \mathrm{dL})$ & 50 & (89) & 38 & (66) & 0.002 & $4.4[1.6$ to 12$]$ \\
\hline \multicolumn{7}{|l|}{ Miliary chest } \\
\hline$x$-ray & 9 & (16) & 2 & (3) & 0.024 & $5.3[1.1$ to 25$\}$ \\
\hline \multicolumn{7}{|l|}{ Sputum smear } \\
\hline \multirow[t]{2}{*}{ AFB positive } & 18 & ( $36 \%$ of & 10 & $(36 \%$ of & & \\
\hline & & 50 patients) & & 56 patients) & 0.034 & $2.6[1.1$ to 6.3$]$ \\
\hline Cough $>1$ month & 26 & (46) & 41 & $(69)$ & 0.009 & $0.37 \mid 0.17$ to $0.79 \mid$ \\
\hline Haemoptysis & 4 & (7) & 11 & (19) & 0.057 & $0.32[0.1$ to 1.1$]$ \\
\hline Classical CXR & 17 & $(30)$ & 31 & (53) & 0.010 & $0.37[0.01$ to 0.87 \\
\hline \multicolumn{7}{|l|}{ Pleural effusion } \\
\hline on CXR & 7 & (12) & 19 & (33) & 0.009 & $0.43 \mid 0.2$ to 0.93 ] \\
\hline \multicolumn{7}{|l|}{ Cavitation on } \\
\hline CXR & 9 & (16) & 10 & (17) & 0.834 & $0.9\left[\begin{array}{lll}0.3 & 10 & 2.4\end{array}\right]$ \\
\hline
\end{tabular}

\section{Discussion}

We found $M$. tuberculosis to be the commonest cause of BSI among febrile adults admitted to a medical ward in an area endemic for HIV and TB, and identified the clinical indicators of mycobacteraemia. The prevalence of mycobacteraemia was much higher than in other African studies [5,6,8], perhaps due to the higher rate of HIV infection in this study. In common with others, non-tuberculous mycobacteraemia was rare.[19,20]

Most $(77 \%)$ patients with mycobacteraemia were identified with routinely available diagnostic methods. Only $11 \%$ of those with mycobacteraemia ( $1.7 \%$ all study patients) were neither detected by routine methods nor entered into a therapeutic trial. In only two people $(0.6 \%$ study patients) did mycobacterial blood culture result in treatment of TB not previously diagnosed. Given the enormous difficulties in following up patients in this setting, mycobacterial blood cultures are unlikely to contribute importantly to routine care.

One potential bias is that clinicians involved in the study may have been more likely to start TB treatment than in routine practice. However we worked to protocols, and 59\% diagnoses were confirmed micobiologically. Only twelve diagnoses were based solely on a 'non-classical' $x$-ray plus symptoms (the most subjective category). If TB is diagnosed less often in routine practice our results suggest that many cases are missed.

Mycobacteraemic patients admitted to the TB ward for short course chemotherapy had a short-term survival at least as good as non-mycobacteraemic TB patients given the same treatment. Their mortality after one month $(21 \%)$ is similar to other series of TB patients $[21,22]$, and compares well with that of patients with miliary TB in the pre-AIDS era, of whom $20 \%$ died within two weeks. [13] We only have data for the first month but this is the period when most deaths occur.[13,22] Although in some patients mycobacteraemia may occur as an acute agonal event [19], in many it is a treatable condition.

Patients selected for a diagnostic trial of TB treatment, on the basis of persistent unexplained fever, were extremely likely to have mycobacteraemia. However five of six died during the trial of treatment (the sixth dying 2 weeks later), so the diagnostic value was in the indications for a trial, not the trial itself. Rifampicin may reduce mortality in such patients [19], although its broad anti-bacterial spectrum means that a response does not confirm TB. Given that the clinical picture correlated so well with mycobacteraemia it seems reasonable to treat these patients promptly with rifampicin based short course chemotherapy.

In this study $11 \%$ of patients diagnosed with $\mathrm{TB}$ had a miliary pattern on chest $x$-ray, compared with only $1 \%$ in another series of TB patients in Malawi.[22] We investigated fever rather than chronic cough, but it suggests that disseminated TB is often missed. We believe that a high index of suspicion and a low threshold for investigating for TB with chest $\mathrm{x}$-ray and sputum smear would improve detection usefully, even without facilities for mycobacterial blood culture. This would allow prompt treatment and a reduction in mortality.

$M$. tuberculosis is the commonest blood stream infection in febrile adults admitted to our hospital. In most, TB is clinically detectable using routinely available investigations, and outcome if treated with rifampicin-based chemotherapy is as good as that in other bacteraemias or in TB without mycobacteraemia. Strengthening of basic facilities (radiology, microscopy for $\mathrm{AFBs}$, and clinical care) would be likely to improve detection and treatment of mycobacterial disease.

\section{Acknowledgements}

This study was funded by grants from the Malawi Health Support Fund of the Royal Netherlands Embassy in Lusaka (grant number MW004401/02), the National Tuberculosis Programme of Malawi, and the Netherlands Tuberculosis Association (KNCV). This paper is a shortened and modified version of the paper which was published in the International Journal of Tuberculosis and Lung Disease 2002; 6: 1067-1074 - we are grateful for editorial permission to publish this version in the $M M J$.

We are grateful to Prof. AD Harries for helpful advice in planning and writing 
the study, and to Ledson Mkwaira, Freda Nsamala, Mercy Mtegha, Gabriel Matheyu, Jonathan Waluza, and to the laboratory technicians at the College of Medicine and Wellcome Trust Research Laboratories. Thanks to Mrs Leen Rigouts and Prof. F Portaels at the Institute for Tropical Medicine, Antwerp, Belgium, who performed the PCR on samples culture-positive for mycobacteria. MEM and ALW are supported by a Research Leave Fellowship from the Wellcome Trust, London, UK.

\section{References}

1. Clough MC. The cultivation of tubercle bacille from the circulation blood in miliary tuberculosis. Am Rev Tuberc 1917; 1: 598-621.

2. Barnes PF, Arevalo. Six cases of Mycobacterium tuberculosis bacteraemia. J Inf Dis $1987 ; 156: 377-8$

3. Boula E, Diaz-Lopez MD, Moreno S, Bernaldo de Quiros JCL, Vicente T, Berenguer J. Mycobacterium tuberculosis bacteraemia in patients with and without Human Hmmunodeficiency Virus infection. Arch Int Med 1993; 153:496-500.

4. Grinsztejn B, Fandinho FC, Veloso VG et al. Mycobacteraemia in patients with the acquired immunodeficiency syndrome. Arch Intern Med. 1997; 157(20): 2359-63.

5. Archibald LK, den Dulk MO, Pallangyo KJ, Reller LB. Fatal Mycobacterium taberculosis bloodstream infections in febrile hospitalized adults in Dar es Salaam, Tanzania. Clin Infect Dis. 1998; 26(2): 290-6.

6. Ssali FN, Kamya MR, Wabwire Mangen F et al. A prospective study of communityacquired bloodstream infections among febrile adults admitted to Mulago Hospital in Kampala, Uganda. J Acquir Immune Defic Syndr Hum Retrovirol. 1998; 19(5): 484-9.

7. McDonald LC, Archibald LK, Rheanpumikankit S et al. Unrecognised Mycobacterium tuberculosis bacteraemia among hospital inpatients in less developed countries. Lancet 1999; 354: 1159-63.

8. Archibald LK, McDonald LC, Nwanyanwu $O$ et al. A hospital-based prevalence survey of bloodstream infections in febrile patients in Malawi: implications for diagnosis and therapy. J Infect Dis 2000; 181: 1414-20.

9. Harries AD. Unrecognised Mycobacterium tuberculosis (letter). Lancet 2000; 355: 142 .

10. Hargreaves NJ, Phiri S, Kwanjana $J$, Nyangulu DS, Squire SB. Unrecognised Mycobacterium tuberculosis (letter). Lancet 2000; 355: 141.

11. Shafer RW, Goldberg R, Sierra M, Glatt AE. Frequency of Mycobacterium tuberculosis bacteraemia in patients with tuberculosis in an area endemic for AIDS Am Rev Respir Dis 1989;140:1611-1613.

12. Vugia DJ, Kjehlbauch JA, Yeboue $\mathrm{K}$ et al. Pathogens and predictors of fatal septicemia associated with human immunodeficiency virus infection in lvory Coast, west Africa. J Infect Dis 1993; 168(3): 564-70.

13. Maartens G. Wilcox PA, Benatar SR. Miliary tuberculosis: rapid diagnosis, hematologic abnotmalities, and outcome in 109 treated adults. Am J Med 1990; 89: 291-296.

14. Chapman CB, Whorten CM. Acute generalised miliary tubereulosis in adults, $\mathrm{N}$ Eng J Med 1946; 235: 239-48

15. Harries AD, Hargreaves NJ, Kumwenda J, Kwanjana JH, Salaniponi FM. Trials of anti-tuberculosis treatment in areas of high immunodeficiency virus prevalence in sub-Saharan Africa. Int J Tub Lung Dis 2000;4:998-1001.

16. Bissuel F, Leport C, Perronne C, Longuet P, Vilde JL. Fever of unknown origin in HIV-infected patients: a critical analysis of a retrospective series of 57 cases. J Int Med 1994; 236: 529-35.

17. Fandinho FCO, Grinsztejn B, Veloso VG et al. Diagnosis of disseminated mycobacterial infection: testing a simple and inexpensive method for use in developing countries. Bulletin WHO 1997;75:361-366.

18. World Health Organisation. WHO case definitions for AIDS surveillance in adults and adolescents. Weekly Epidem Rec 1994; 69: 273-5.

19. Arthur G, Nduba VN, Kariuki SM, Kimari J, Bhatt SM, Gilks CF. Trends in bloodstream infections for HIV-infected adults admitted to hospital in Nairobi, Kenya over the last decade. Clin Infect Dis 2001;33:248-256

20. Gilks CF, Brindle RJ, Mwachari C et al. Disseminated Mycobacterium avium infection among HIV-infected patients in Kenya. J Acquir Immune Def Syndr 1995; $8: 195-198$

21. Harries AD, Nyirenda TE, Bannerjee A, Boeree MJ, Salaniponi FML. Treatment outcome of patients with smear-negative and smear-positive pulmonary uberculosis in the National Tuberculosis Control Programme, Malawi. Trans Roy Soc Trop Med Hyg 1999; 93: 443-446.

22. Harries AD, Nyangulu DS, Kang'ombe C et al. Treatment outcome of an unselected cohort of tuberculosis patients in relation to human immunodeficiency virus serostatus in Zomba hospital, Malawi. Trans Roy Soc Trop Med Hyg 1998; 92 : 343-7.

23. Harries AD. Tuberculosis in Africa: clinical presentation and management. Pharmacol Ther 1997; 73: 1-50.

24. Elliott AM, Nkando L, Tembo G et al. Impact of HIV on tuberculosis in Zambia: a cross sectional study. Br Med J 1990; 301: 4i2-5.

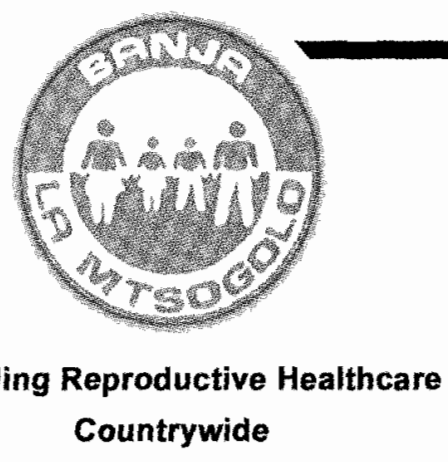

\title{
Providing Reproductive Healthcare \\ Countrywide
}

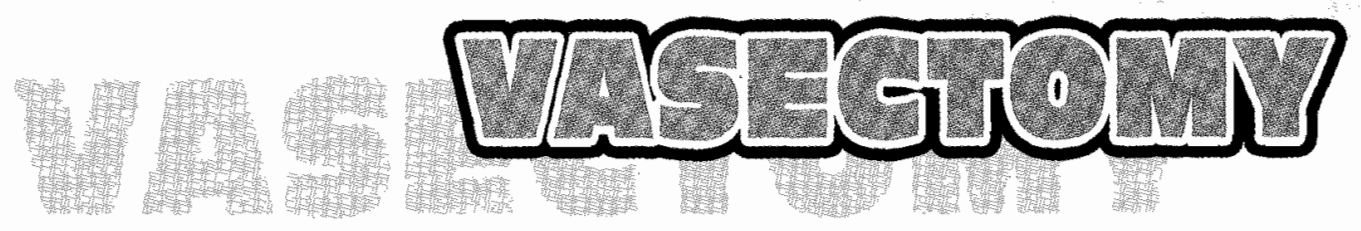

\author{
The permanent family planning method \\ for men, like medical practitioners, available in \\ all BLM Clinics. For men who really love \\ their spouses
}

Visit the nearest BLM Clinic today or call $01673844 / 240$ for more information

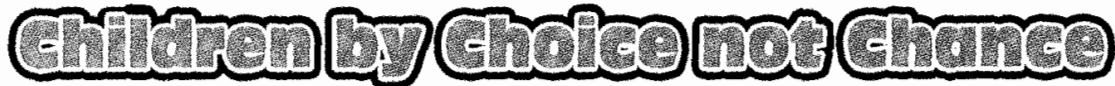

